\title{
Estudo comparativo entre limites radiográfico e histológico em ameloblastomas*
}

\section{Comparative study among radiographic and histological limits in ameloblastomas}

Heloísa Emília Dias da Silveira** João Jorge Diniz Barbachan ***

\section{RESUMO}

Com o objetivo de observar a presença e comparar a distância, entre as infiltrações de células neoplásicas no osso e o limite radiográfico em ameloblastomas, foram selecionadas seis peças cirúrgicas enviadas ao Laboratório de Patologia Bucal da UFRGS, que apresentavam-se íntegras e com os limites do tumor radiograficamente identificáveis, sendo estas submetidas a exames radiográficos e cortes histológicos seriados. A partir dos resultados obtidos podemos concluir que, apesar do ameloblastoma apresentar radiograficamente limites definidos é histologicamente infiltrativo. Estas infiltrações ocorrem em poucos e distintos locais, variando, neste estudo, de $0,1 \mathrm{~cm}$ a $1,4 \mathrm{~cm}$ da extremidade radiográfica do neoplasma.

\section{UNITERMOS}

Ameloblastoma; patologia bucal; tumores odontogênicos

\section{INTRODUÇÃO E REVISÃO DA LITERATURA}

0 ameloblastoma é um tumor originário de tecido epitelial odontogênico que, desde as suas primeiras descrições na literatura, tem sido objeto de constantes estudos, exercendo um fascínio especial sobre clínicos, radiologistas, cirurgiões e patologistas. A literatura sobre o assunto é volumosa e, paradoxalmente, uma fonte de informações conflitantes, principalmente quanto à conduta terapêutica a ser adotada que, indiscutivelmente é cirúrgica, embora outros tratamentos tenham sido testados ao longo do tempo. A discussão que se mantém ainda hoje se refere ao tipo de abordagem cirúrgica, e isto se deve ao fato de o tumor mostrar limites radiográficos definidos e apresentar histologicamente um comportamento infiltrativo (EBLING, 1977 ; MÜLLER e SLOOTWEG, 1985; MACINTOSH, 1991). Este fato acaba levando os cirurgiões a assumirem condutas de tratamento em relação ao ameloblastoma de acorda com o praticado em suas instituições de ensino (GOLD, 1991).

Segundo DEMEULEMEESTER et al. (1988) o problema da recidiva existe pela inadequada ressecção do osso medular envolvido, difusão de fragmentos ósseos residuais com tecido tumoral e, principalmente, por uma excisão cirúrgica imperfeita dos tecidos moles adjacentes. $\mathrm{O}$ índice de recidiva é alto nos casos tratados conservadoramente conforme observado por SEHDEV et al. apud BREDENKAMP et al. (1989), UENO et al.(1989), OLAITAN et al.(1998) e SAMPSON e POGREL(1999).

$\mathrm{O}$ ameloblastoma apresenta um crescimento localmente invasivo e, por isso, o tratamento padrão descrito na literatura é a ressecção cirúrgica com $1 \mathrm{~cm}$ de margem óssea de segurança. Entretanto, esta distância varia, segundo os autores, de $0,5 \mathrm{~cm}$ (SHATKIN e HOFFMEISTER, 1965), 1,5 - 2cm (MACINTOSH, 1991), a 3cm (GOLD, 1991). Foi nosso objetivo, neste trabalho, realizar um estudo comparativo entre o limite radiográfico e o limite histológico em ameloblastomas, procurando verificar a existência e a distância das infiltrações neoplásicas nas margens que circundam o tumor. Para tanto, nos propusemos a:

1. Observar a presença de infiltrações de células neoplásicas no osso além dos limites apresentados radiograficamente pelo tumor;

2. Medir a distância entre o limite radiográfico do neoplasma e as invasões celulares tumorais no osso adjacente.

\section{MATERIAL E MÉTODOS}

Seleção das peças cirúrgicas: Das peças operatórias de ameloblastomas encaminhadas ao Laboratório de Patologia da Faculdade de Odontologia da Universidade Federal do Rio Grande do Sul, forma selecionadas seis, que pela sua integridade permitiam a avaliação entre o limite da lesão e o osso não envolvido radiograficamente.

Execução das radiografias: As peças foram radiografadas no seu sentido vestíbulo-lingual em filme $18 \times 24$, com $40 \mathrm{~cm}$ de distância foco-objeto para se eliminar distorção radiográfica. Utilizou-se aparelho de $R x$ odontológico com $70 \mathrm{kVp}-10 \mathrm{~mA}$ e tempo de exposição de 0,3 segundos.

Realização da histotécnica: $\mathrm{O}$ material foi descalcificado em ácido nítrico a 5\%, com controle diário, até permitir a secção com bisturi, nas zonas escolhidas a partir do exame radiográfico (limite da lesão observável circundado por osso radiograficamente não comprometido). Realizou-se então, processamento histotécnico de rotina para inclusão em parafina e coloração em hematoxilina-eosina. Os cortes semi-seriados de $5 \mathrm{~mm}$ foram executados no sentido próximo-proximal, iniciando pela vestibular, com intervalos de $50 \mathrm{~mm}$. A quantidade de blocos confeccionados a partir de cada peça não foi igual, já que estes provieram de áreas onde era possível verificar a extremidade radiográfica do tumor e o osso adjacente. Estes foram numerados segundo a numeração da peça e da ordem em que foram confeccionados, e ainda, quando subdivididos em superior e inferior, em relação a peça, ao lado do número foi colocada a letra $\mathrm{S}$ ou I.

A partir das seis peças cirúrgicas utilizadas para a realização deste trabalho, foram confeccionadas 1092 lâminas histológicas derivadas de 45 blocos de parafina.

Medição das infilttrações tumorais: As medições foram realizadas nas lâminas, examinadas ao microscópio, desde a borda tumoral até o ponto mais distante onde eram encontradas células neoplásicas. Este ponto era então marcado com caneta especial para

\footnotetext{
** Profa. Adjunta da Faculdade de Odontologia da UFRGS, Porto Alegre, RS. Especialista em Radiologia Odontológica/UFSC, Mestre em Patologia Bucal/ UFRGS, Doutora em Estomatologia Clínica/ PUCRS.

*** Prof. Titular da Faculdade de Odontologia da UFRGS, Porto Alegre, RS. Doutor em Patologia Bucal.

* Trabalho apresentado na XII Reunião Anual da SBPqO.
} 
retroprojetor e, posteriormente, medido com régua milimetrada. A seguir, as mensurações obtidas eram repassadas para as radiografias.

Variações nas medições realizadas: Apesar de sabermos da existência de uma contração macroscópica em torno de $20 \%$ para material incluído em parafina, observamos que esta contração somente ocorreu em alguns casos. Isto se deve, provavelmente, ao fato de que o material ficou por períodos variados no ácido cuja temperatura sofreu alteração ao longo do ano. Houve também variação no tamanho da peça e no tipo e quantidade de osso (basal ou medular) envolvido. Quando esta contração era observada, a mesma era considerada nas medições realizadas.

\section{RESULTADOS}

Peça 5017/79. A partir desta peça, foram confeccionados 11 blocos de parafina que originaram 291 lâminas histológicas, oriundos dos locais onde observamos o limite do tumor e osso não envolvido radiograficamente. Trata-se de um ameloblastoma do tipo multilocular, onde estão envolvidos corpo e ramo da mandíbula, com exceção do processo coronóide e côndilo. Praticamente toda a peça se encontra comprometida pela neoplasia (figura 1). A tabela 1 apresenta o resultados encontrados.

Peça 8039/89. Corresponde a um ameloblastoma do tipo multilocular, cuja peça cirúrgica representa uma secção de osso cortical mandibular (figura 2). Foram confeccionados 2 blocos, derivados das extremidades mesial e distal da peça, onde se observava limite e área não envolvida radiograficamente pelo tumor. Estes deram origem a 47 lâminas histológicas. A tabela 2 apresenta os resultados encontrados.

Peça 9113/91. Trata-se de um ameloblastoma do tipo unilocular, cuja peça cirúrgica corresponde ao corpo da mandíbula desde a região de pré-molares e ramo, incluindo processo coronóide e côndilo (figura 3). Foram confeccionados 16 blocos, e, a partir destes, 382 lâminas histológicas. A tabela 3 apresenta os resultados encontrados.

Peça 9826/92. A peça cirúrgica é um segmento da mandíbula apresentadá pela região de molares e 2 pré-molar. O ameloblastoma é do tipo multilocular (figura 4). Desta peça, foram confeccionados seis blocos, resultando em 147 lâminas histológicas. A tabela 4 apresenta os resultados encontrados.

Peça 9884/93. Esta peça corresponde a um ameloblastoma do tipo multilocular e representa um segmento de mandíbula que engloba desde região de incisivo lateral até ramo com exceção do processo coronóide e côndilo (figura 5). Foram confeccionados quatro blocos de parafina derivados das extremidades mesial e distal da peça, selecionados pelas áreas que interessavam radiograficamente, já que o tumor comprometia praticamente todo o osso. Dos quatro blocos de parafina, foram confeccionadas 72 lâminas histológicas. A tabela 5 apresenta os resultados encontrados.

Peça 10156/93. Corresponde a um ameloblastoma do tipo multilocular, cuja peça cirúrgica compreende corpo de mandíbula desde a região de pré-molares, envolvendo o ramo, processo coronóide e.côndilo (figura 6). Foram confeccionados seis blocos de parafina, a partir das extremidades da peça, que resultaram em 153 lâminas histológicas. A tabela 6 apresenta os resultados encontrados.

Ao se analisar os resultados obtidos, verifica-se que $40 \%$ dos blocos confeccionados resultaram em lâminas histológicas que demonstraram células tumorais, enquanto que $60 \%$ destes derivaram cortes que nada apresentaram. As infiltrações neoplásicas observadas variaram de $0,10 \mathrm{~cm}$ a $1,40 \mathrm{~cm}$ além do limite radiográfico do ameloblastoma. Torna-se interessante ressaltar, ao se verificar os dados encontrados, que, em muitos casos, blocos oriundos de áreas justapostas ao tumor ou em contato direto com este radiograficamente derivaram lâminas histológicas que não acusaram a presença de células neoplásicas.

\section{DISCUSSÃO}

A conduta cirúrgica em relação ao tratamento do ameloblastoma é muito discutida e apresenta opiniões variadas, principalmente em relação à margem de segurança a ser adotada. Isto se deve ao fato de o tumor exibir limites radiográficos definidos e apresentar histologicamente um comportamento infiltrativo (MÜLLER e SLOOTWEG, 1985; MACINTOSH, 1991).

Considerando-se estas características próprias da patologia, se torna fácil compreender o alto índice de recidiva encontrado em casos tratados conservadoramente (SEHDEV et al. apud BREDENKAMP et al., 1989; UENO et al., 1989; OLAITAN et al., 1998; SAMPSON e POGREL, 1999).

Para DEMEULEMEESTER et al. (1988) o problema da recidiva deve-se à ressecção inadequada do osso medular envolvido, à difusão de fragmentos ósseos residuais com tecido tumoral e, acima de tudo, a uma excisão cirúrgica imperfeita dos tecidos moles adjacentes.

A maioria dos autores defende a ressecção cirúrgica com margem de segurança para o ameloblastoma sólido ou multicístico. Esta margem é geralmente distante 1 a $2 \mathrm{~cm}$ do limite radiográfico do tumor.

No entanto, GOLD (1991) defende que a distância mínima confiável para a margem de segurança seria de $3 \mathrm{~cm}$, alegando não ter conhecimento, com base comprovada, nos limites adotados de menor comprimento.

MACINTOSH (1991) observou ninhos de tecidos ameloblástico quase $1 \mathrm{~cm}$ além da margem histológica do neoplasma principal e sustenta que a linha de ressecção deve ser de $1,5 \mathrm{~cm}$ e, preferencialmente, $2 \mathrm{~cm}$ além do palpável ou do limite radiográfico da lesão.

Para SHATKIN e HOFFMEISTER (1965) a margem de tecido saudável necessária para garantir uma excisão completa é de 0,5 a $1 \mathrm{~cm}$ de osso normal a volta do tumor, e uma margem similar em mucosa ao redor do sítio da biópsia.

Analisando os resultados obtidos neste trabalho, na avaliação de existência e distância de infiltrações neoplásicas nas margens que circundam o tumor, podemos observar que estas existem e distaram de 0,1 a $1,4 \mathrm{~cm}$ da extremidade radiográfica do ameloblastoma. A maioria das células tumorais presentes estavam localizadas junto à borda da lesão ou distavam desta aproximadamente $0,2 \mathrm{~cm}$.

Assim, considerando os dados por nós obtidos, concordamos com MACINTOSH (1991), que defende uma linha de ressecção preferencialmente a $2 \mathrm{~cm}$ do limite radiográfico do tumor.

Ao se examinar os elementos encontrados, torna-se evidente alguns resultados muito interessantes.

Das seis peças cirúrgicas analisadas, apenas uma apresentou infiltração, e em um só local, maior que $1 \mathrm{~cm}(1,4 \mathrm{~cm})$. Nas demais a presença de células neoplásicas se fez a poucos milímetros da extremidade tumoral e em três destas (9113/91, 9826/92 e 10156/93) não ultrapassou dois locais, mostrando uma infiltração tumoral pouco esparsa. Isto talvez explique o motivo pelo qual, raras vezes, e ainda levando-se em consideração o fator sorte, se obtenha sucesso com tratamento conservador, que, para ser comprovado, necessita de muitos anos de, acompanhamento, segundo EBLING (1977), a vida toda.

Outro achado curioso foi o fato de vários blocos terem sido confeccionados a partir de locais onde observávamos radiograficamente tecido ósseo não comprometido e área neoplásica e, no entanto, não encontramos presenças de células tumorais. Isto provavelmente ocorreu porque nestas áreas havia apenas estroma da lesão, com suas características histológicas inespecíficas, e não parênquima.

Segundo UENO et al. (1989), recidivas em ameloblastomas tratados conservadoramente foram muito mais frequientes naqueles que apresentavam imagem radiográfica multilocular (60-70\%), se comparadas aos tipos unilocular (35\%).

Em nosso trabalho, a infiltração neoplásica mais longínqua do limite radiográfico 
do tumor $(1,4 \mathrm{~cm})$ ocorreu em um caso de ameloblastoma unilocular, o que tornaria interessante um estudo posterior sobre o assunto.

É imprescindível ainda salientar que, em duas peças cirúrgicas, tivemos células tumorais encontradas no limite extremo das mesmas, o que nos leva a imaginar a existência de infiltração além do que encontramos.

\section{CONCLUSÕES}

Os resultados encontrados em nosso estudo nos permitem concluir que:

1. O ameloblastoma, apesar de apresentar radiograficamente limites bem definidos, histologicamente é infiltrativo, e estas invasões celulares neoplásicas exibem distâncias variadas;

2. A infiltração tumoral, geralmente, se encontra a poucos milímetros dos limites da extremidade radiográfica do neoplasma $\mathrm{e}$, muitas vezes, em poucos e distintos locais, mostrando uma invasão pouco dispersa;

3. A presença de células tumorais mais distantes das bordas radiográficas da lesão que encontramos foi a $1,4 \mathrm{~cm}$ dos mesmos, nos levando a sugerir uma margem de segurança cirúrgica de $2 \mathrm{~cm}$ além dos limites observados na radiografia.

\section{KEYWORDS}

Ameloblastomal; Odontogenic tumors; Buccal pathology.

\section{SUMARY}

The aim of this study was to analyse the presence of histological infiltrations of neoplastic cells within the bone, and compare the distance of these infiltrations with the radiografic limits of the ameloblastoma. For this purpose, six integral surgical samples, with identifiable radiographic limits of the tumor, sent to the Pathology Service of Dental School of the Federal University of Rio Grande do Sul, were processed. From the results obtained, can be concluded that, although ameloblastoma presents well-defined radiographic limits, it is infiltrative from the histological view point. Infiltrations occurs at few and distinctive sites, varying in this study from $0.10 \mathrm{~cm}$ to $1.40 \mathrm{~cm}$, related to the radiographic borders of the neoplasm.

\section{REFERÊNCLAS BIBLIOGRÁFICAS}

$$
\text { 1. BREDENKAMP, }
$$

J.K.;

ZIMMERMAN, M.C.; MICKEL, R.A. Maxillary ameloblastoma: a potentially lethal neoplasm. Arch. Otolaryngol. Head Neck Surg., Chicago, v. 115, p. 99-104, Jan. 1989.

2. DEMEULEMEESTER, L.J.-K.J. et al. Late loco-regional recurrences after radical resection for mandibular ameloblastoma. Int. J. Oral Maxillofac. Surg., Copenhagen, v. 17 , n. 5 , p. $310-315$, Oct. 1988 .

3. EBLING, H. Cistos e tumores odontogênicos. 3. ed., Porto Alegre: UFRGS; McGraw Hill do Brasil, 1977, p. 39-82.

4. GOLD, L. Biologic behavior of ameloblastoma. Oral Maxillofac Surg. Clin. North Am., Philadelphia, v. 3, n. 1, p. 2127, Feb. 1991.

5. MACINTOSH, R.B. Agressive surgical management of ameloblastoma. Oral
Maxillafac. Surg. Clin. North Am., Philadelphia, v. 3, n. 1, p. 73-97, Feb. 1991.

6. MÜLLER, H,; SLOOTWEG, P.J. The ameloblastoma, the controversial approach to therapy. J. Maxillofac. Surg., Philadelphia, v. 13, n. 2, p. 79-84, Apr. 1985.

7. OLAITAN, A.A.; AROLE, G.; ADEKEYE, E.O. Recurrent ameloblastoma of the jaws. A follow-up study. Int. J. Oral Maxillofac. Surg., Copenhagen, v. 27, n. 6, p. 456-460, Dec. 1998.

8. SAMPSON, D.E.; POGREL, M.A. Management of mandibular ameloblastoma: the clinical basis for a treatment algorithm. J. Oral Maxillofac. Surg., Copenhagen, v. 57, n. 9, p. 1074-1079, Sept. 1999.

9. SHATKIN, S.; HOFFMEISTER, F.S. Ameloblastoma: a rational approach to therapy. Oral Surg. Oral Med. Oral Pathol., St. Louis, v. 20, n. 4, p. 421-435, Oct. 1965.

10 UENO, S.; MUSHIMOTO,

K.; SHIRASU, R. Prognostic evaluation of ameloblastoma based on histologyc and radiographic typing. J. Oral Maxillofac. Surg., Philadelphia, v. 47, n. 1, p. 11-15, Jan. 1989.

Endereço para Correspondência:

Heloísa E. Dias da Silveira

Rua Ramiro Barcelos, 2492

0-XX-51- 316.5199

e-mail: silvebona@cpovo.net

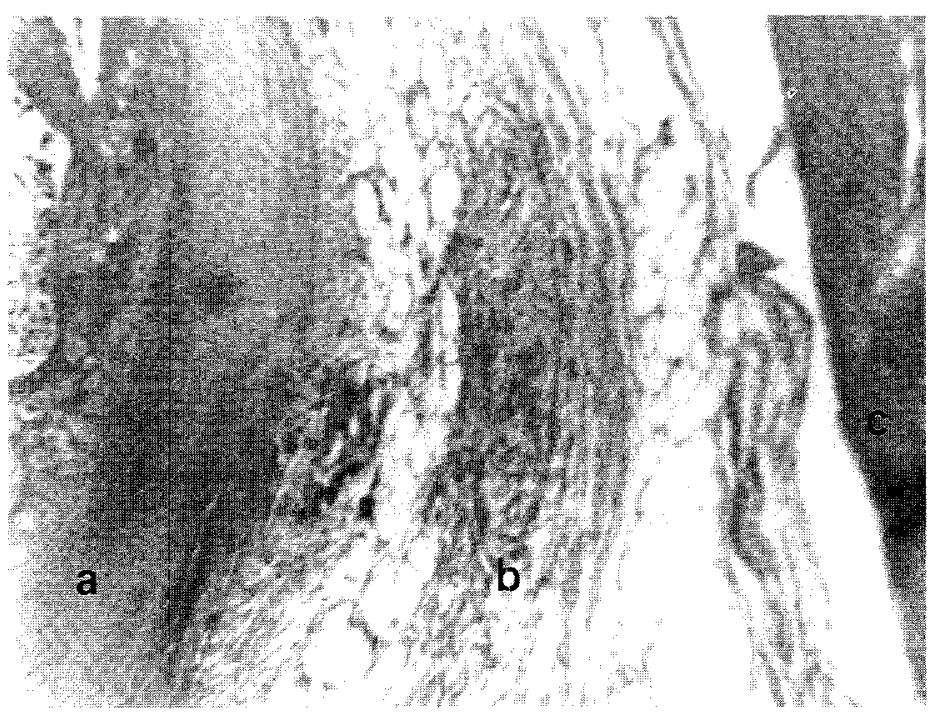

Figura 1-Aspecto radiográfico da peça 5017/79. As áreas pontilhadas indicam onde foram encontradas as infiltraçõs tumorais nos blocos 3 e 4 da mesma.

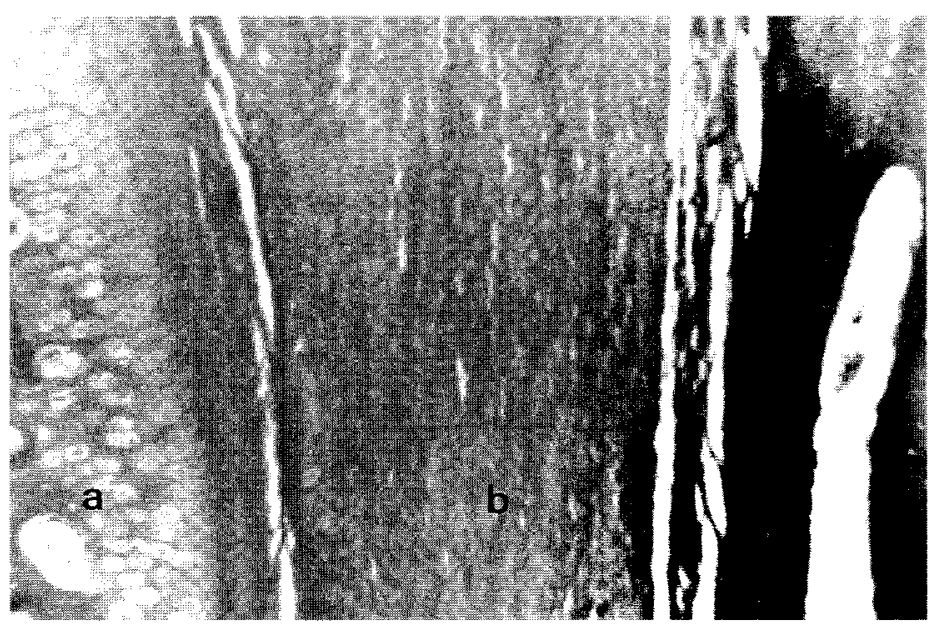

Figura 2- Aspecto radiográfico da peça 8039/89. As áreas pontilhadas indicam onde foi observado histologicamente presença de células neoplásicas. 


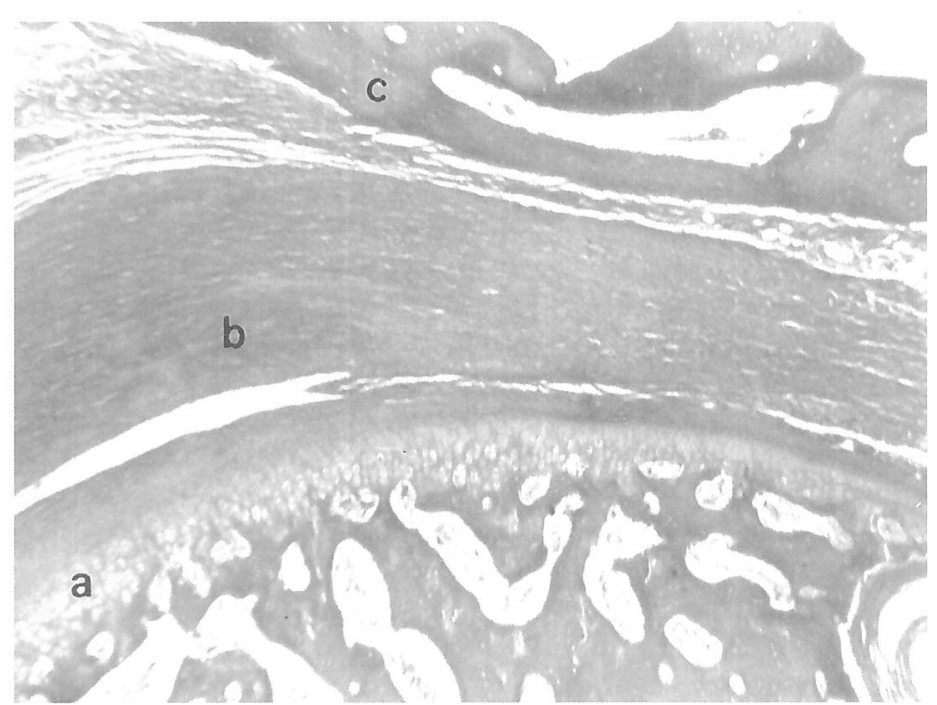

Figura 3-Aspecto radiográfico da peça 9113/91. As áreas pontilhadas nas regiões correspondentes ao bloco 1 II e 5 indicam onde foi observado histologicamente a presença de infiltração de células neoplásicas.

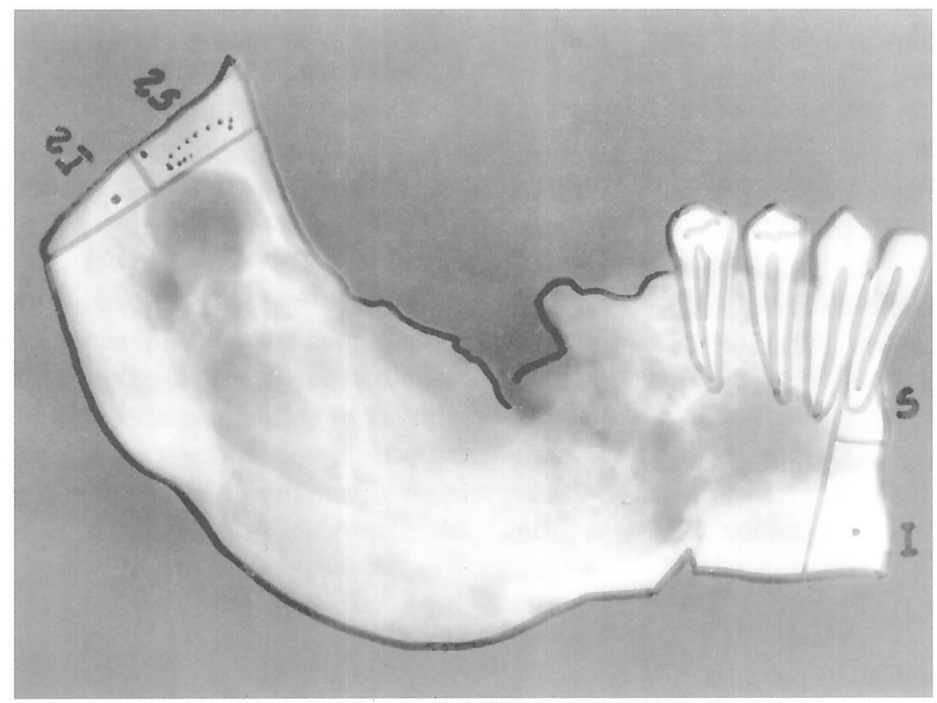

Figura 5- Aspecto radiográfico da peça 9884/93. As áreas pontilhadas indicam onde foi observado histologicamente a presença de células neoplásicas.

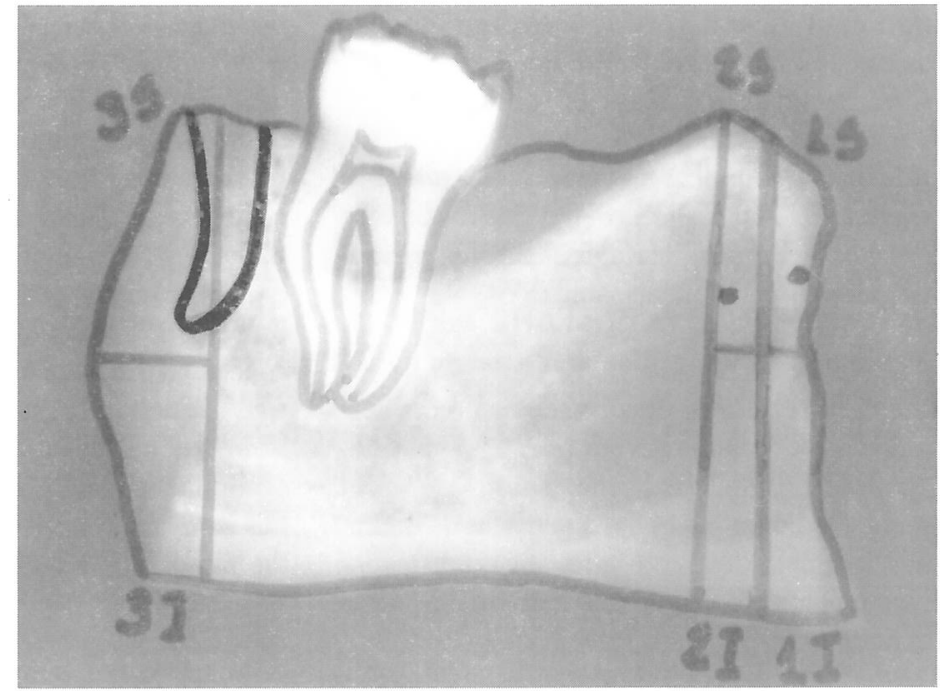

Figura 4- Aspecto radiográfico da peça 9826/92. Os pontos marcados nas áreas correspondentes aos blocos 9826/92 IS e 9826/92 2S (parte superior distal da peça) indicam onde foi observado histologicamente a presença de células neoplásicas.

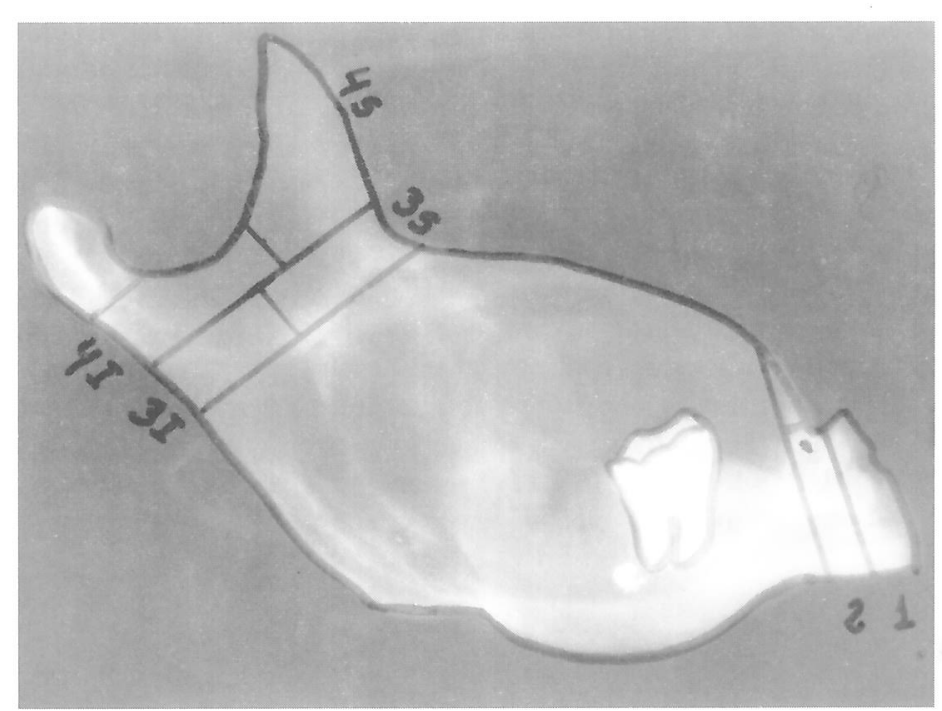

Figura 6- Aspecto radiográfico da peça 10156/93. O ponto na área correspondente ao bloco $10156 / 932$ indica onde foi encontrado infiltração tumoral. 
TABELA 1 - Infiltrações na peça 5017/70

\begin{tabular}{c|c|c}
\hline Número do bloco & Presença de células tumoriais & $\begin{array}{c}\text { Distância do limite } \\
\text { radiográfico do tumor }\end{array}$ \\
\hline $5017 / 793-1$ & Sim & Junto ao limite \\
\hline $5017 / 793-2$ & Sim & Junto ao limite \\
\hline $5017 / 793-3$ & Sim & Junto ao limite \\
\hline $5017 / 793-4$ & Sim & $0,20 \mathrm{~cm}$ \\
\hline $5017 / 793-5$ & Sim & $0,20 \mathrm{~cm}$ \\
\hline $5017 / 793-6$ & Sim & $0,16 \mathrm{~cm}$ \\
\hline $5017 / 793-7$ & Não & - \\
\hline $5017 / 794-1$ & Não & Junto ao limite \\
\hline $5017 / 794-2$ & Sim & Junto ao limite \\
\hline $5017 / 794-8$ & Sim & - \\
\hline $5017 / 794-9$ & Não & \\
\hline
\end{tabular}

TABELA 2 - Infiltrações na peça $8039 / 89$.

\begin{tabular}{c|c|c}
\hline Número do bloco & Presença de células tumoriais & $\begin{array}{c}\text { Distância do limite } \\
\text { radiográfico do tumor }\end{array}$ \\
\hline $8039 / 891$ & Sim & Junto ao limite \\
\hline $8039 / 892$ & Sim & $0,25 \mathrm{~cm}$ \\
\hline
\end{tabular}

TABELA 1 - Infiltrações na peça $5017 / 70$

\begin{tabular}{c|c|c}
\hline Número do bloco & Presença de células tumoriais & $\begin{array}{c}\text { Distância do limite } \\
\text { radiográfico do tumor }\end{array}$ \\
\hline $9113 / 91$ IS & Não & - \\
\hline $9113 / 91$ II & Sim & - \\
\hline $9113 / 912 \mathrm{~S}$ & Não & - \\
\hline $9113 / 91$ 2I & Não & - \\
\hline $9113 / 913 \mathrm{~S}$ & Não & - \\
\hline $9113 / 913 \mathrm{I}$ & Não & - \\
\hline $9113 / 914$ & Não & - \\
\hline $9113 / 915$ & Sim & - \\
\hline $9113 / 916$ & Não & - \\
\hline $9113 / 917$ & Não & - \\
\hline $9113 / 918 S$ & Não & - \\
\hline $9113 / 918 \mathrm{I}$ & Não & - \\
\hline $9113 / 919 \mathrm{~S}$ & Não & - \\
\hline $9113 / 919 \mathrm{I}$ & Não & - \\
\hline $9113 / 9110 \mathrm{~S}$ & Não & \\
\hline $9113 / 9110 \mathrm{I}$ & Não & \\
\hline
\end{tabular}


TABELA 4 - Infiltrações na peça 9826/92.

\begin{tabular}{c|c|c}
\hline Número do bloco & Presença de células tumoriais & $\begin{array}{c}\text { Distância do limite } \\
\text { radiográfico do tumor }\end{array}$ \\
\hline $9826 / 921 \mathrm{~S}$ & Sim & $0,10 \mathrm{~cm}$ \\
\hline $9826 / 92$ 1I & Não & - \\
\hline $9826 / 922 \mathrm{~S}$ & Sim & Dentro da área tumoral \\
\hline $9826 / 92$ 2I & Não & - \\
\hline $9826 / 923 \mathrm{~S}$ & Não & - \\
\hline $9826 / 923 \mathrm{I}$ & Não & - \\
\hline
\end{tabular}

TABELA 5 - Infiltrações na peça 9884/93.

\begin{tabular}{c|c|c}
\hline Número do bloco & Presença de células tumoriais & $\begin{array}{c}\text { Distância do limite } \\
\text { radiográfico do tumor }\end{array}$ \\
\hline $9884 / 93$ IS & Não & - \\
\hline $9884 / 93$ 1I & Sim & $0,20 \mathrm{~cm}$ \\
\hline $9884 / 932 \mathrm{~S}$ & Sim & $0,50 \mathrm{~cm}$ \\
\hline $9884 / 93$ 2I & Não & $0,30 \mathrm{~cm}$ \\
\hline
\end{tabular}

TABELA 6 - Infiltrações na peçà $10156 / 93$.

\begin{tabular}{c|c|c}
\hline Número do bloco & Presença de células tumoriais & $\begin{array}{c}\text { Distância do limite } \\
\text { radiográfico do tumor }\end{array}$ \\
\hline $10156 / 931$ & Nào & - \\
\hline $10156 / 932$ & Sim & $0,20 \mathrm{~cm}$ \\
\hline $10156 / 933 \mathrm{~S}$ & Não & - \\
\hline $10156 / 933 \mathrm{I}$ & Não & - \\
\hline $10156 / 934 \mathrm{~S}$ & Não & - \\
\hline $10156 / 934 \mathrm{I}$ & Não & - \\
\hline
\end{tabular}

